\title{
An Ultra-Fast 10Gb/s 64b66b Data Serialiser Back- end in 65nm CMOS Technology
}

\author{
Thomas Gardiner ${ }^{1}$ \\ Science and Technology Facilities Council \\ Rutherford Appleton Laboratory, Harwell Oxford, Didcot OX11 OQX, UK \\ E-mail: Thomas.Gardinerastfc.ac.uk
}

\begin{abstract}
With future pixel ASICs trending towards mega-frame rate readout, the development of ultrahigh-speed readout systems is increasingly important. Here we present an ultra-fast readout system developed to operate at $10 \mathrm{Gbps}$, and intended to surpass a more conventional highlyparallel LVDS bus approach. The system generates a $5 \mathrm{GHz}$ clock (LC Oscillator), scrambles and serialises the parallel input data in accordance with the Aurora 64b66b protocol, and transmits the data off-chip through a Current Mode Logic (CML) line-driver at 10Gbps. A prototype is under evaluation having been fabricated in early 2018 on a $65 \mathrm{~nm}$ Multi-Project Wafer. Serialiser ASIC ran at 10.312Gbps under test for 60 hours without a bit-error event.
\end{abstract}

Topical Workshop on Electronics for Particle Physics (TWEPP2018)

17-21 September 2018

Antwerp, Belgium

1

Thomas Gardiner 


\section{Introduction}

Future pixel ASICs are now trending towards readout speeds in the mega-framerate order. This has put considerable strain on traditional readout systems and has resulted in a significant increase in area, power and IO pads. These readout systems typically function on a simple interface scheme and LVDS drivers to transmit the data off chip. This work has developed a new sophisticated readout system with a significant increase in link speed and a large reduction in area, power and IO pads. Furthermore, an interface module is proposed and is in development to maintain a low-complexity interface despite the large internal complexity increase.

Figure 1 shows the top-level hierarchy of the 10Gbps serialiser. The serialiser interface in development is to be asynchronous; externally interfacing data is timed to an unconstrained clock generated elsewhere in the ASIC, while internally a $5 \mathrm{GHz}$ clock generated from a LC oscillator PLL is the governing clock domain for all internal data. Once the data is processed and transferred to the $5 \mathrm{GHz}$ clock domain, data is encoded and scrambled according the Aurora 64b66b protocol. Data is then split, mixed and serialized into a $10 \mathrm{Gbps}$ data stream to be transmitted off chip through a differential CML driver.

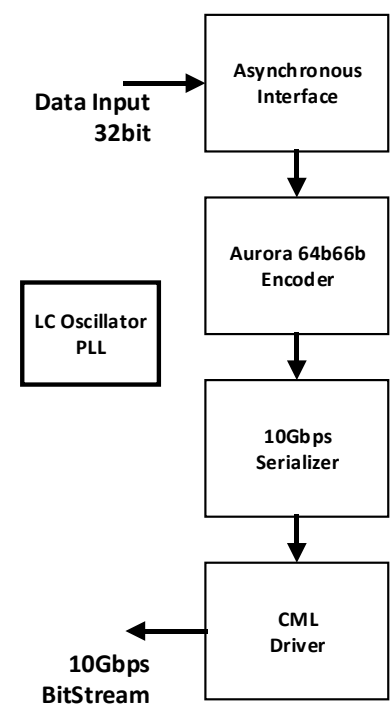

Figure 1: 10Gbps Serialiser Readout System.

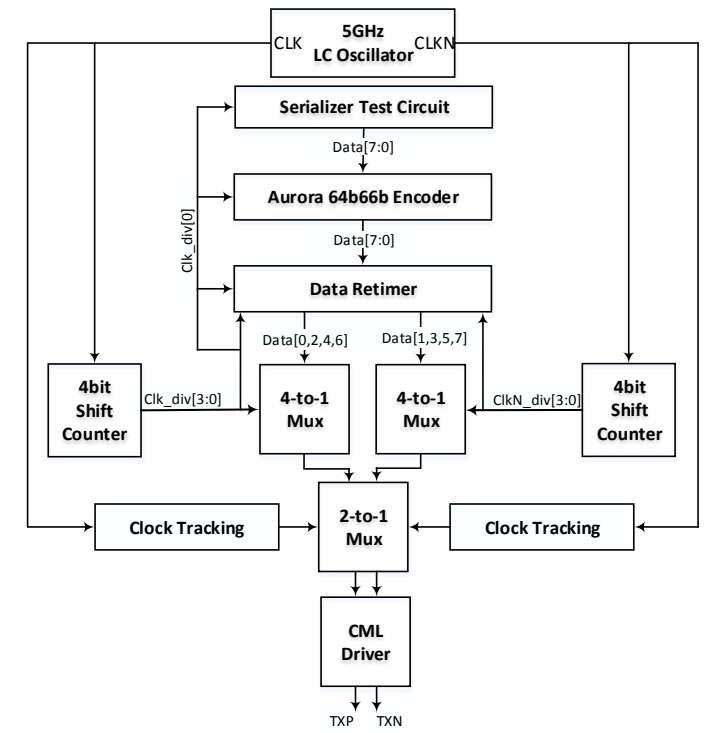

Figure 2: 10Gbps Serialiser Architecture.

\section{Serialiser Architecture}

The serialiser architecture is shown in Figure 2 and compromises of a 5GHz PLL module, Interface logic module, $10 \mathrm{Gbps}$ Serialiser and CML Driver. The final output of this module produces an Aurora 64b66b compliant 10Gbps bitstream and is transmitted off chip.

\subsection{LC Oscillator PLL}

An LC oscillator was chosen for its superior performance in jitter, duty cycle and stability. Additional switchable damping capacitors were added to provide flexibility to the target frequency. The LC oscillator was attached to an analogue charge pump PLL architecture to 
control the frequency and stability of the clock. Dynamic TSPC flip-flops were implemented to divide the $5 \mathrm{GHz}$ clock down for comparison with the reference clock $(\div 128)$. The TSPC flipflops were required to allow state-based digital circuitry to operate at $5 \mathrm{GHz}$, these flip-flops were also used in the serialiser circuit [1]. Figure 3 and Figure 4 show the schematic of the TSPC flip-flop and the LC oscillator respectively.

\subsection{Gbps Serialiser}

The serialiser has been designed to accept 8-bit words per clock cycle (which operates at $1.25 \mathrm{GHz})$ at the input and output a differential full-swing10Gbps bitstream.

\subsubsection{Interface Scheme}

The serialiser module has been designed to operate asynchronously with the remainder of the ASIC hence an asynchronous FIFO transfer module is in development. 32bit words are written asynchronously and read out in 8bit words using standard clock domain crossing techniques and elastic buffers. Status flags such as FIFO_empty are used to automatically send idle packets when no written data is available.

\subsubsection{Aurora 64b66b Encoding}

Aurora 64b66b protocol was chosen due to being a robust and common protocol besides contains many high-level features besides scrambling the data to ensure a DC balanced bitstream for clean data-transmission. Features implemented in this design include: 1) Clock recovery packets - Allows the receiver to keep the clock locked to the data stream, 2) End frame packets - Allows the bit-stream to be packaged automatically at the receiver end and 3) Idle packets - Allows idle padded data to be automatically stripped out at the output of the receiver.

The encoder circuit encodes 8 bits from the input and outputs the previously encoded 8 bit word at the output. Since the protocol adds 2 header bits every 64 bits to form a 66bit data packet, the encoder circuit contains an elastic shift buffer to store the remaining bits. When 8 remainder bits have been accumulated, the encoder raises an output to indicate that the 8 bit input word will not be accepted and instead proceeds to output the 8 remaining bits hence flushing the elastic buffer; this occurs every 4 encoded data packets. Note that this does not distort the bitstream order as the remaining bits are replaced every cycle with the 8bit input word. It's worth mentioning that the protocol requires all 64 bits to be streamed in consecutive cycles and only on the last streamed in 8bit word can the interface module decide whether to send an idle or end-frame packet or to continue sending data. In the test circuit, the interval of idle and end-frame packets is programmable.

\subsubsection{8-to-1 bit Serialiser}

The 8 -to- 1 bit serialiser circuit operates at $1.25 \mathrm{GHz}$ (generated from the $5 \mathrm{GHz}$ clock) and encodes and serialises 8 bits per cycle to form the 10Gbps bit stream. The 8bit words are encoded following the Aurora 64b66b protocol as described above before being serialised.

Firstly, the encoded 8 bit word is retimed with each individual bit being consecutively shifted by 100ps to ensure a single valid bit is available at all times. Precise time shifting is achieved by the generation of eight $1.25 \mathrm{GHz}$ clocks using two 4bit shift counters with each counter being updated on opposite clock edges of the $5 \mathrm{GHz}$ clock. The eight different clocks 
then retime the 8 bit word by passing each bit safely through 1 or 2 different clock domains before finishing on the predestined clock domain. The retimed 8bit word is then split in two alternately and fed into two 4-to-1 serialisers to create two 5Gbps bitstreams. The two 5Gbps bitstreams are finally multiplexed into a single $10 \mathrm{Gbps}$ bitstream with a 2-to-1 multiplexer controlled by the phased-tracked $5 \mathrm{GHz}$ clock.

\subsection{Current Mode Line-Driver}

The Current Mode Line-Driver (CML) was implemented using a standard architecture as shown in Figure 5. Charge balancing transistors (half width transistors) were added to correct the charge injected from the driver transistors due to a large gate to drain capacitance.

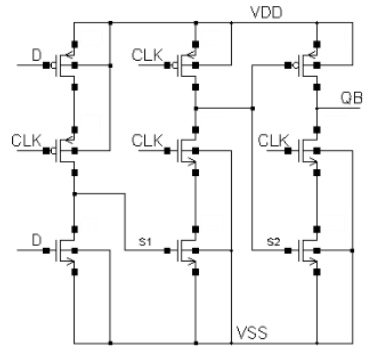

Figure 3: Schematic of Figure 4: Schematic of LC Oscillator. TSPC Flip-flop.

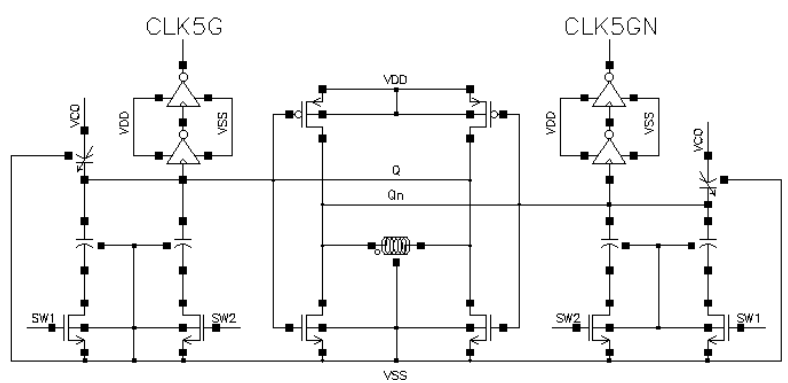

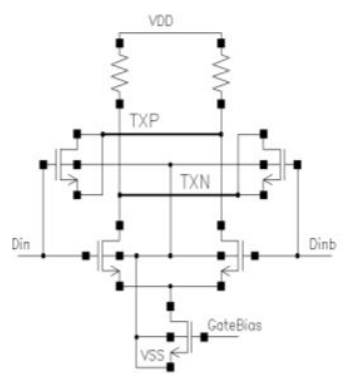

Figure 5: Schematic of CML Driver.

\subsubsection{Link Modelling}

To ensure the CML driver generated a clean eye off chip, the link was modelled in 3 parts: wire-bond, PCB and termination IC models and simulated in a spice simulation. Wire-bond model consisted of an RLC network and was derived from first principles due to being well understood. The PCB model was an extracted model of the link tracks taken from an early design of the test board with manufacturing properties included. The termination IC model contained loading capacitance and termination impedance.

\section{Testing}

The sophisticated test system includes the following modules: 1) A retimer chip (TI DS110DF410) to sample the 10Gbps bitstream and buffer it off the PCB. 2) A Xilinx Kintex-7 FPGA with a 10Gbps Aurora 64b66b transceiver synthesized internally along with error detection and data capture code for evaluating the quality and robustness of the ASIC serialiser, 3) A programmable crystal (Si570) to generate a clean stable clock for the ASIC PLL, 4) A Clock multiplier (Si5324) to generate a compatible clock for the Kintex-7 transceiver at the defined $10.302 \mathrm{Gbps}$ bit-rate.

Once the system is initialized, an encoded bitstream containing a continuous PRBS-11 sequence using Aurora 64b66b protocol is sent from the ASIC to the FPGA where the PRBS-11 sequence is recovered. The synthesized error detection and data capture code checks the incoming bit sequence for long periods of time and records when bit errors occur. Figure 6 shows the difference between modelled and measured result. The degradation of the eye opening is likely to be caused by jitter noise in the PLL, non-idealities in the link model and 
measurement probe distortion. Figure 7 shows a large eye opening for a very low Bit Error Rate (BER) when resampling at the retimer IC.

a)

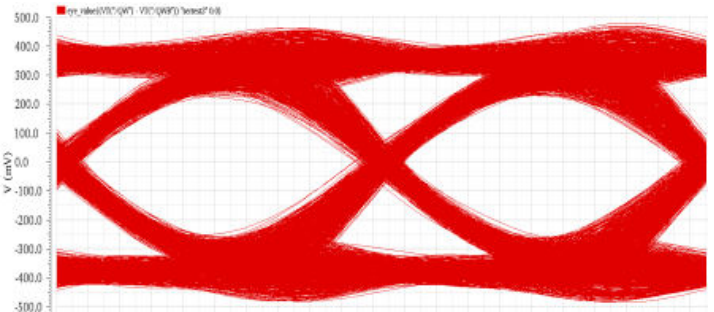

b)
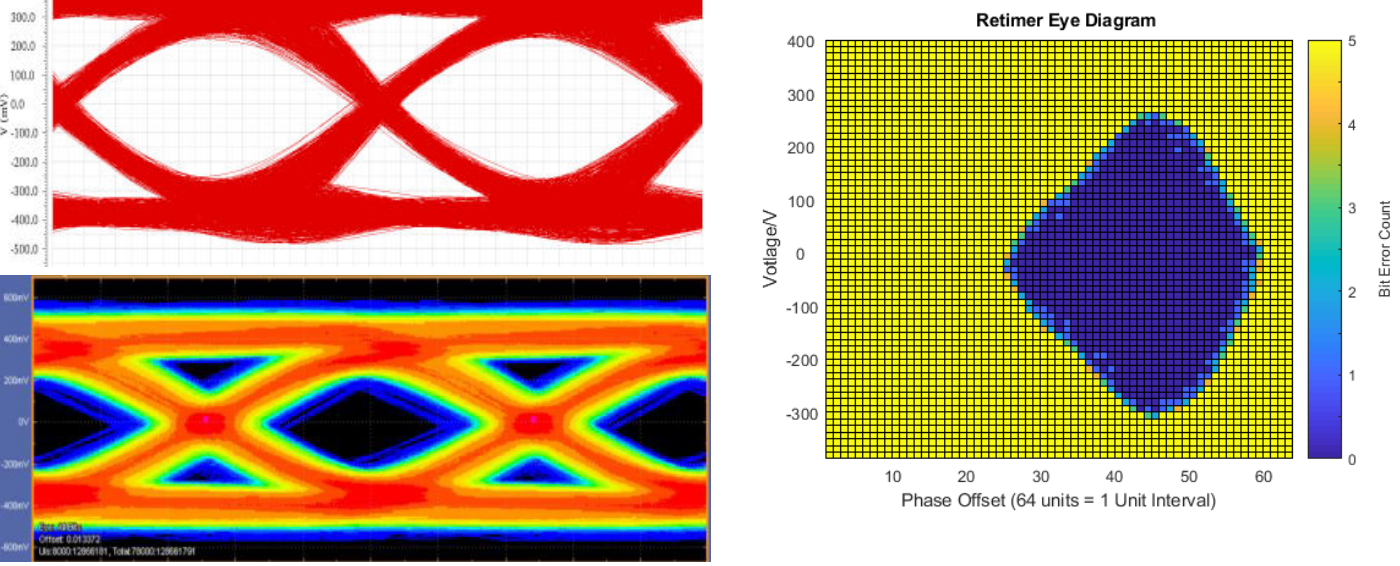

Figure 6: a) Simulated 10Gbps bitstream with an eye opening amplitude of $400 \mathrm{mV}$ and width of 80ps. b) Measured 10Gbps bitstream with an eye opening of $300 \mathrm{mV}$ and $45 \mathrm{ps}$.

Figure 7: Detected 10Gbps bitstream eye diagram at the input of the Retimer IC. Recorded by the Retimer IC's debug circuitry.

\section{Conclusion}

In this paper, a $10.302 \mathrm{Gbps}$ serialiser readout system has been designed and tested successfully with a BER of $10^{-16}$. Once the low-complexity interface finishes development, the readout system will be easily deployable onto ASIC designs due to its self-contained nature. Figure 8 shows the assembled system under test and Figure 9 shows the physical high-speed link.

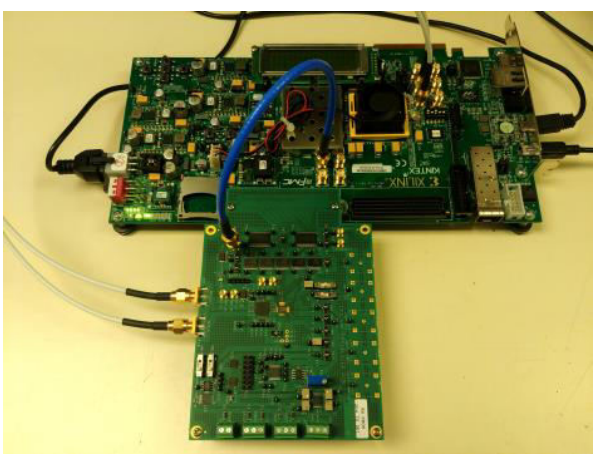

Figure 8: Readout system in operation with test board and FPGA board.

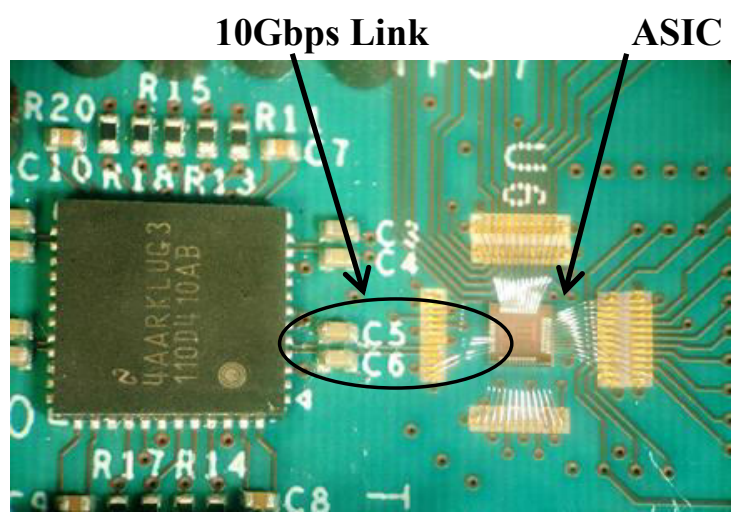

Figure 9: ASIC wire-bonded to test board with the high-speed link highlighted.

\section{References}

[1] D. Felici, S. Bertazzoni, S. Bonacini, A. Marchioro, P. Moreira and M. Ottavi, A $20 \mathrm{~mW}, 4.8 \mathrm{Gbit} / \mathrm{sec}$, SEU robust serializer in $65 \mathrm{~nm}$ for read-out of data from LHC experiments, 2014 JINST 9 C01004. 